\begin{tabular}{|c|c|c|}
\hline $\begin{array}{l}\text { PKS } \\
\text { PUBLIC } \\
\text { KNOWLEDGE } \\
\text { DNOLIECT }\end{array}$ & $\begin{array}{c}\text { Revista de GEOGRAFIA } \\
\text { (RECIFE) } \\
\text { http://www.revistauffe.bbr revistageografia }\end{array}$ & $\begin{array}{l}\text { OJS } \\
\text { OPEN } \\
\text { JOURNAL } \\
\text { CYCTEMS }\end{array}$ \\
\hline
\end{tabular}

\title{
APLICAÇÕES E PERSPECTIVAS DO SENSORIAMENTO REMOTO PARA O MAPEAMENTO DE ÁREAS INUNDÁVEIS
}

\author{
Osmar Abílio de Carvalho Júnior ${ }^{1}$ \\ ${ }^{1}$ Universidade de Brasília.Email:osmarjr@unb.br
}

Artigo recebido em 13/08/2018 e aceito em 14/09/2018

\begin{abstract}
RESUMO
O sensoriamento remoto permite a obtenção de informações consistentes, atualizadas e relativamente precisas para o monitoramento de corpos hídricos, auxiliando nas atividades de conservação e manejo das áreas úmidas, análise de inundações, modelos de previsão de desastres, entre outros. O presente artigo apresenta uma revisão das inovações tecnológicas do sensoriamento remoto aplicadas ao monitoramento de áreas inundáveis. Recentemente, essa área do conhecimento atravessa por diversas transformações com expressivo impacto na aquisição e processamento de dados. Portanto, a revisão foca nos estudos desenvolvidos com sensoriamento remoto radar e óptico para a detecção da superfície d'água e discute as perspectivas do advento de novas tecnologias, tais como: processamento em nuvens, imagens com alta resolução temporal e novos algoritmos para o processamento dos dados. O processamento em nuvem permite um acesso a coleções volumosas de imagens orbitais de observação da Terra para um grande número usuários, o que era até pouco tempo inviável. O advento das constelações de nanossatélites transforma completamente o processo de aquisição das imagens, que resulta na diminuição significativa dos custos, obtenção de dados diários e com alta resolução espacial. A conjunção desses fatores proporciona a aceleração de troca de conhecimento e converge para uma observação global integrada.
\end{abstract}

Palavras-chaves: Computação em Nuvem, Satélite Miniaturizado, Alta Resolução Temporal, Imagem de Satélite, Inundações.

\section{APPLICATIONS AND PERSPECTIVES OF REMOTE SENSING FOR WETLAND MAPPING}

\begin{abstract}
Remote sensing allows consistent, up-to-date and relatively accurate information for monitoring of water bodies, assisting in the conservation and management of wetlands, flood analysis, disaster prediction models, among others. This paper presents a review of the technological innovations in remote sensing applied to the monitoring of flooded areas. Recently, this field of knowledge presents several changes with a significant impact on the acquisition and processing of data. Therefore, the review focuses on the studies developed with radar and optical remote sensing for the detection of water surface and discusses the perspectives of new technologies, such as cloud processing, high temporal resolution images and new algorithms for data processing. Cloud processing enables access to full collections of Earth observation images to a large number of users, which was unfeasible until recently time. The advent of nanosatellite constellations completely transforms the image acquisition process, resulting in significant cost savings, daily data acquisition, and high spatial resolution. The combination of these factors provides the acceleration of knowledge exchange and converges to an integrated global observation.
\end{abstract}

Keywords: Cloud Computing, Small Satellite, High Temporal Resolution, Satellite imagery, Floods. 


\section{INTRODUÇÃO}

As imagens de sensoriamento remoto orbital permitem uma aquisição de informações sistemáticas, sinópticas e com baixos custos da superfície da Terra, auxiliando a compreensão dos intrincados sistemas naturais e antrópicos. Esses dados permitem uma reconstrução da história recente sendo fundamental para o mapeamento e formulação de modelos da hidrosfera, atmosfera, biosfera e da dinâmica humana (SILVA et al., 2012). Especificamente em estudos geomorfológicos, dados de sensores remotos são utilizadas para o mapeamento e previsão de diferentes processos, tais como: inundação e dinâmica fluvial (KLEMAS, 2014; RAHMAN \& DI, 2017), dinâmica de dunas (HERMAS et al. 2012; HUGENHOLTZ et al., 2012), erosões (MATA et al., 2007; LANE et al., 2003; VRIELING, 2006), movimento de massa (GUIMARÃES et al., 2008, METTERNICHT et al., 2005; SCAIONI et al. 2014; TOFANI et al., 2013), detecção de feições cársticas (CARVALHO JUNIOR et al., 2008, 2013; SIART et al., 2009), geomorfologia glacial (KÄAB et al., 2008), entre outros. Assim, informações geoespaciais derivados de sensoriamento remoto são cada vez mais usados em sistemas de apoio à decisão para a definição de políticas públicas, gestão territorial, monitoramento ambiental e sistemas de alertas de desastres em diversas escalas (TRALLI et al., 2005; VAN WESTEN, 2013). As séries temporais de imagens de satélite permitem avaliar os fatores de risco e de potencial presentes dentro de um ambiente natural ou antropizado, visando uma adequação de uso, exploração, conservação, mitigação e priorização de respostas.

O contínuo desenvolvimento e sofisticação dos sensores remotos e das infraestruturas de armazenamento e gerenciamento de dados convergem para uma observação global integrada, considerando os diferentes atributos climáticos, hidrológicos, geomorfológicos e humanos. Recentemente, os principais adventos tecnológicos no sensoriamento remoto são: (a) a alta disponibilização de imagens de satélite de acesso aberto; (b) desenvolvimento de captação de imagens por nanossatélites; (c) processamento em nuvem baseada em supercomputadores e sistemas de computação de alta performance (por exemplo o Google Engine); (d) disponibilidade de várias ferramentas desenvolvidas em código livre para o processamento de dados geoespaciais, e (d) elaboração de modelos digitais de alta resolução usando Interferometria SAR, Lidar e fotogrametria digital. A conjunção desses fatores acelera o conhecimento e aplicação do sensoriamento remoto, aumentando as investigações nas diversas escalas espaciais e temporais com aquisição de dados condizentes com os ciclos naturais. Além disso, um número maior de usuários consegue efetuar 
integrações volumosas de dados orbitais de observação da Terra, que eram inviáveis até pouco tempo (HIRD et al., 2017).

O presente trabalho possui como objetivo revisar os principais avanços tecnológicos na área do sensoriamento remoto e mostrar sua aplicação para estudos de monitoramento da dinâmica de inundações fluviais. Nesse propósito, a revisão é subdividida nos seguintes temas: revisão das pesquisas no mapeamento de áreas inundáveis a partir de sensoriamento remoto; computação em nuvem e sua implicação no mapeamento da água superficial, e o advento dos nanossatélites no monitoramento da cobertura terrestre.

\section{MAPEAMENTO DE ÁREAS INUNDÁVEIS}

A precisa distribuição espacial dos corpos d'água são dados fundamentais para gestão e monitoramento dos recursos hídricos (SAWAYA et al., 2003); inventário de áreas úmidas (OZESMI \& BAUER, 2002; GALLANT et al., 2015); modelos hidráulicos (MUSA et al., 2015; YAN et al., 2015); previsão de enchentes (SCHUMANN et al., 2015; GRIMALDI et al., 2016); e monitoramento e avaliação de risco de desastres (SMITH, 1997). As imagens de sensoriamento remoto permitem descrever a distribuição espacial das águas superficiais terrestres, que seria impossível com a simples expansão in situ das redes de estações fluviométricas. A evolução dos métodos e análises de dados de sensoriamento remoto para estudos hídricos torna-se cada vez mais rápida devido à disponibilidade de extensos conjuntos de dados e algoritmos livres (JONES et al., 2009). Assim, uma grande variedade de imagens está disponível para o mapeamento de zonas úmidas tanto por sensores do tipo radar como óptico, considerando diferentes resoluções espaciais, espectrais e temporais.

Para o monitoramento do pulso hidrológico um importante quesito é a resolução temporal devido à alta variabilidade sazonal do nível d'água nas zonas úmidas. As mudanças da dinâmica da água são monitoradas a partir de uma série temporal com alto ciclo de periodicidade, gerando um cubo 3D temporal com as dimensões espaciais nos eixos " $x$ " e " $y$ " e a dimensão temporal no eixo "z" (Figura 1).

O método de mapeamento de áreas inundáveis mais simples é a aplicação de um fatiamento da imagem por um valor limite, sendo computacionalmente rápido e com obtenção de bons resultados para imagens ópticas e de radar. Nas imagens ópticas, normalmente é efetuado o cálculo de um índice espectral antes do fatiamento para evidenciar e distinguir as áreas de superfície de água. No entanto, uma limitação desse método é a necessidade de uma calibração de acordo com a região de estudo, uma vez que os limites são dependentes do 
conteúdo de água e das propriedades espectrais da região, o que impede de ser um método completamente automatizado (COLTIN et al., 2016).

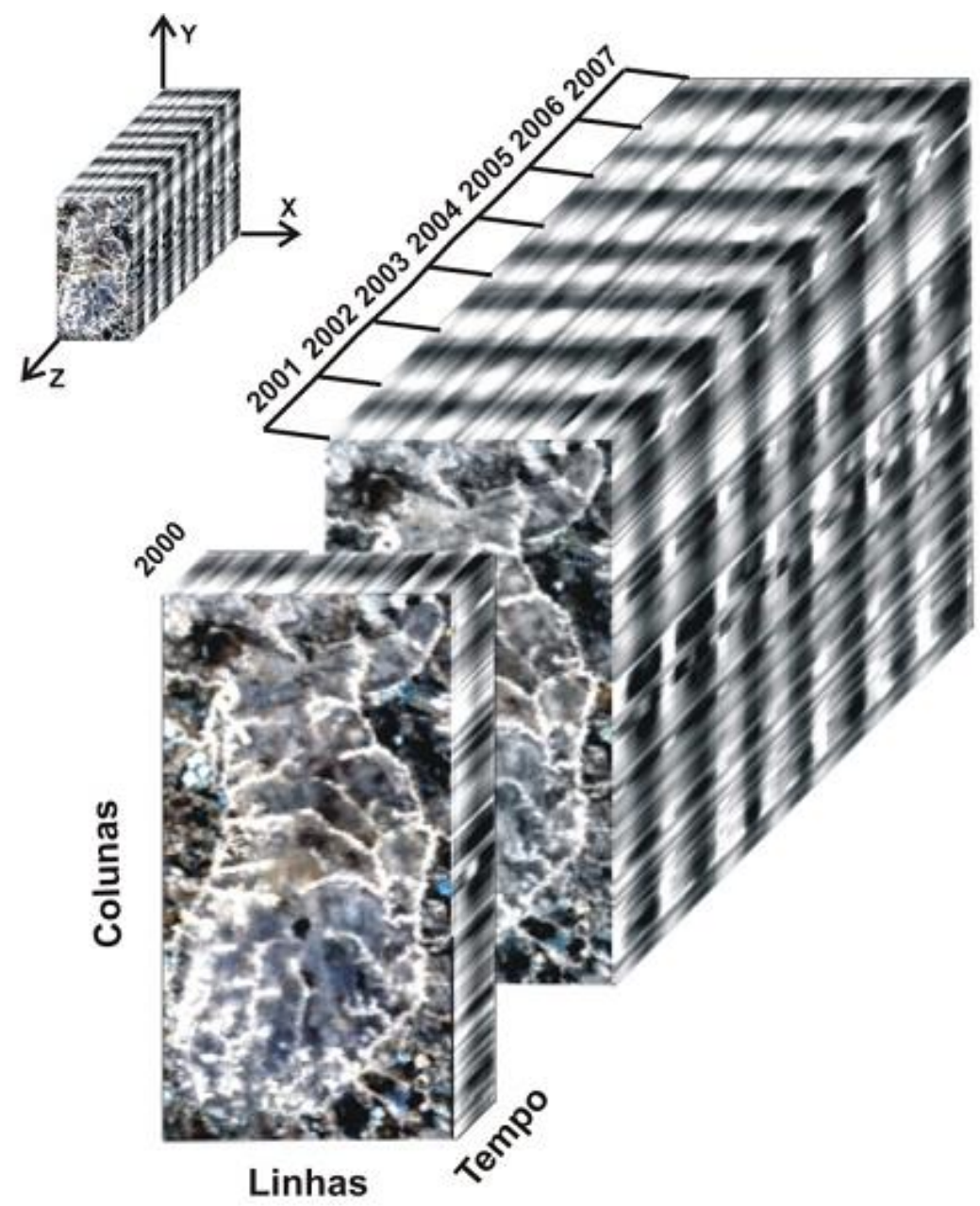

Figura 1. Cubo temporal de imagens NDVI MODIS do Campo de Instrução de Formosa, Goiás (Carvalho Júnior et al., 2009).

Nessa abordagem, diferentes índices espectrais normalizados são usados em imagens ópticas para a detecção de água, tais como (Tabela 1): Normalized Difference Water Index (NDWI) (MCFEETERS, 1996); Modified Normalized Difference Water Index (MNDWI) (XU et al., 2006); Normalized Difference Moisture Index (NDMI também conhecido como $\mathrm{NDWI}_{\mathrm{GAO}}$ ) (GAO et al. 1996; WILSON et al., 2002); Water Ratio Index (WRI) (SHEN et al., 2010); Land Surface Water Index (LSWI) (XIAO et al., 2004); Normalized Difference Vegetation Index (NDVI) (ROUSE et al., 1973, DOMENIKIOTIS et al., 2003; ROGERS \& KEARNEY, 2004); Enhanced Vegetation Index (EVI) (HUETE et al., 2002, XIAO et al., 2006); Normalized Difference Flood Index 1 e 2 (NDFI1 e NDFI2) (BOSCHETTI et al., 
2014) e Floating Algae Index (FAI) (HU et al., 2009; FENG et al. 2012). Estudos comparativos entre os índices espectrais normalizados apontam para uma melhor performance do índice MNDWI (BOSCHETTI et al., 2014; JI et al., 2009; LI et al. 2013).

Além disso, alguns autores propõem formulações contendo associação de índices espectrais. Xiao et al., (2006) utiliza conjuntamente valores de diferença entre LSWI e EVI e (DVLE) e entre LSWI e NDVI (DVLN). Islan et al., (2010) desenvolve uma árvore de decisão considerando os índices EVI, LSWI e DVLE. Índices mais complexos são também propostos como o Automated Water Extraction Index (AWEI) que utiliza cinco bandas espectrais do Landsat 5 TM para aumentar o contraste e acurácia entre a água e outras superfícies escuras (FEYISA et al., 2014). Nessa abordagem, foram desenvolvidas duas formulações (Tabela 1): (a) $\mathrm{AWEI}_{\text {nsh }}$ elaborado para eliminar efetivamente os pixels não aquáticos, incluindo as superfícies escuras de áreas urbanas; e (b) $\mathrm{AWEI}_{\mathrm{sh}}$ que efetua uma melhora na precisão da remoção dos pixels de sombra, onde eventualmente o $\mathrm{AWEI}_{\text {nsh }}$ não proporciona uma eliminação efetivamente. Outro algoritmo que integra diferentes bandas e índices espectrais para a determinação da probabilidade da presença de água é o Open Water Likelihood (OWL) (GUERSCHMAN et al., 2011; CHEN et al. 2013, TICEHURST et al. 2014).

Outro procedimento ainda pouco explorado é o método de fatiamento sobre bandas ou índices espectrais que foram transformadas do espaço de cor RGB (Red-Green-Blue) para o HSI (Hue-Saturation-Intensity) (JIANG et al., 2012) ou HSV (Hue-Saturation-Value) (PEKEL et al., 2014). A transformação usando Análise de Componentes Principais também pode ser aplicada em séries temporais NDWI (NDWI-PCs) para salientar a presença e mudanças dos corpos hídricos (ROKNI et al. 2014). No caso específico do tratamento de imagens hiperespectrais para o mapeamento de vegetações de áreas inundadas, Adam et al. (2010) realizarão uma revisão dos métodos e bandas espectrais mais utilizadas.

Em regiões com alta porcentagem de cobertura de nuvens como a região Amazônica, as imagens ópticas apresentam limitações para discriminar ao longo do tempo as zonas úmidas. A alternativa disponível é o emprego das imagens de radar que fornecem informações em qualquer condição climática e durante o dia e a noite (Rosenqvist et al., 2007). Dessa forma, um grande avanço de pesquisas no bioma Amazônico tem sido realizado a partir de imagens de radar. 
Tabela 1 - Índices espectrais utilizados para a detecção de corpos d'água. Onde IVP é infravermelho próximo, IVM é infravermelho médio, IVOC é infravermelho de ondas curtas. Especificamente para o índice EVI, a variável L é um ajuste do dossel fundo, C1 e C2 são os coeficientes da resistência ao aerossol e G é um fator de ganho.

\begin{tabular}{|c|c|c|}
\hline Índice Espectral & Formulação & Referência \\
\hline $\begin{array}{l}\text { Normalized Difference } \\
\text { Water Index }\end{array}$ & $N D W I=\frac{V e r d e-I V P}{V e r d e+I V P}$ & $\begin{array}{c}\text { McFeeters } \\
\text { (1996) }\end{array}$ \\
\hline $\begin{array}{l}\text { Modified Normalized } \\
\text { Difference Water } \\
\text { Index }\end{array}$ & $M N D W I=\frac{V e r d e-I V M}{V e r d e+I V M}$ & $\begin{array}{l}\text { Xu et al. } \\
(2006)\end{array}$ \\
\hline $\begin{array}{c}\text { Normalized Difference } \\
\text { Moisture Index }\end{array}$ & $M D M I=N D W I_{G A O}=\frac{I V P-I V M}{I V P+I V M}$ & $\begin{array}{c}\text { Gao et al. } \\
\text { (1996) e } \\
\text { Wilson et al. } \\
\quad(2002)\end{array}$ \\
\hline Water Ratio Index & $W R I=\frac{\text { Verde }- \text { Vermelho }}{\text { Verde }+ \text { Vermelho }}$ & $\begin{array}{l}\text { Shen et al. } \\
\text { (2010) }\end{array}$ \\
\hline $\begin{array}{l}\text { Land Surface Water } \\
\text { Index }\end{array}$ & $L S W I=\frac{I V P-I V O C}{I V P+I V O V}$ & $\begin{array}{c}\text { Xiao et al. } \\
\text { (2004) }\end{array}$ \\
\hline $\begin{array}{l}\text { Normalized Difference } \\
\text { Vegetation Index }\end{array}$ & $N D V I=\frac{\text { IVP }- \text { Vermelho }}{I V P+\text { Vermelho }}$ & $\begin{array}{l}\text { Rouse et al. } \\
\text { (1973) }\end{array}$ \\
\hline $\begin{array}{l}\text { Enhanced Vegetation } \\
\text { Index (EVI) }\end{array}$ & $E V I=G \frac{\text { IVP }- \text { Vermelho }}{\left(\text { IVP }+C_{1} \text { Vermelho }-C_{2} \text { Azul }+L\right)}$ & $\begin{array}{l}\text { Huete et al. } \\
\text { (2002) }\end{array}$ \\
\hline $\begin{array}{l}\text { Normalized Difference } \\
\text { Flood Index } 1\end{array}$ & $N D F I_{1}=\frac{\text { Vermelho }-N I R}{\text { Vermelho }+ \text { NIR }}$ & $\begin{array}{l}\text { Boschetti et } \\
\text { al. } 2014\end{array}$ \\
\hline $\begin{array}{l}\text { Normalized Difference } \\
\text { Flood Index } 2 \mathrm{e}\end{array}$ & $\mathrm{NDFI}_{2}=\frac{\text { Vermelho }- \text { IVOC }}{\text { Vermelho }+ \text { IVOC }}$ & $\begin{array}{l}\text { Boschetti et } \\
\text { al. (2014) }\end{array}$ \\
\hline Floating Algae Index & $F A I=I V P-\left(\right.$ Vermelho $+($ IVOC - Vermelho $)\left(\frac{\lambda_{I V P}-\lambda_{\text {Vermeliho }}}{\lambda_{\text {IVOC }}-\lambda_{\text {Vermelho }}}\right.$ & $\begin{array}{l}\text { Hu et al. } \\
\text { (2009) }\end{array}$ \\
\hline $\begin{array}{l}\text { Automated Water } \\
\text { Extraction Index }\end{array}$ & $\begin{array}{c}A W E I_{\text {neh }}=4(\mathrm{Verde}-\mathrm{IVM})-(0,25 \mathrm{IVP}+2,75 \mathrm{IVOC}) \\
A W E I_{\text {sh }}=\mathrm{Azul}+2,5 \mathrm{Verde}-1,5(\mathrm{IVP}+\mathrm{IVM})-0,25 \mathrm{IVOC}\end{array}$ & $\begin{array}{l}\text { Feyisa et al. } \\
\text { (2014) }\end{array}$ \\
\hline $\begin{array}{l}\text { Normalized } \\
\text { Polarization }\end{array}$ & $P O L=\frac{V H-V V}{V H+V V}$ & $\begin{array}{l}\text { Hird et al. } \\
\text { (2017) }\end{array}$ \\
\hline
\end{tabular}

Diversas revisões do emprego das imagens radar para o mapeamento dos corpos d'água e áreas inundadas foram desenvolvidos, sendo destacados os seguintes: Hess et al. (1990), Kasischke et al. (1997), Schmullius and Evans (1997), Ramsey (1998), Di Baldassarre 
et al (2011), Schumann e Moller (2015). No caso específico do emprego de sensoriamento remoto para a região Amazônia, Melack e Hess (2010) realizaram uma extensiva revisão e Hall et al. (2011) fizeram uma revisão da modelagem hidrodinâmica da Amazônia usando sensoriamento remoto.

As ondas de radar em corpos de água lisos e abertos são refletidas para longe da antena, tornando o alvo escuro e relativamente simples de ser detectado. Portanto, o fatiamento com um valor limite nas imagens de radar é também o método preponderante para a detecção de água. Normalmente, o fatiamento é feito sobre a imagem de radar após uma etapa de filtragem para a suavização dos ruídos. Recentemente, Hird et al. (2017) adaptaram o Microwave Polarization Difference Index (MPDI) (CHAUHAN et al., 2016) baseado na relação das polarizações vertical-vertical (VV) e vertical-horizontal (VH) para detecção de água (Tabela 1). Inicialmente, o MPDI foi proposto para salientar a densidade de dossel vegetal é a umidade do solo, mas demonstrou potencial para corpos hídricos.

No mapeamento de áreas úmidas com SAR, a banda-L $(15-30 \mathrm{~cm})$ é predominantemente utiliza devido ao maior comprimento de onda, enquanto as bandas $\mathrm{C}$ $(3,75-7,5 \mathrm{~cm})$ e $X(2,40-3,75 \mathrm{~cm})$ são minoritariamente empregadas por apresentar menor penetração no dossel de vegetação densa. Além do fatiamento, outras técnicas de classificação têm sido aplicadas para o mapeamento de áreas inundáveis: redes neurais (AUGUSTEIJN e WARRENDER, 1998), classificação orientada a objeto (OBIA) (EVANS et al., 2010; SILVA et al., 2010), árvore de decisão (PARMUCHI et al. 2002; WHITCOMB et al., 2009), K-NN (BETBEDER et al., 2014), e Support Vector Machine (BETBEDER et al., 2014, ZHANG et al. 2009). A revisão de Henderson e Lewis (2008) descrevem uma falta de consenso nas técnicas de classificação, onde diferentes métodos foram comparados entre si sem estabelecer um consenso (supervisionados tradicionais, redes neurais, sistemas baseados em conhecimentos e especialistas, índices de dispersão e classificadores hierárquicos). Os trabalhos revisados empregam imagens tanto em número digital como coeficientes de retroespalhamente $\sigma^{0} \mathrm{~dB}$. A mesma falta de conformidade pode ser observada para os filtros utilizados para minimizar os efeitos do ruído speckle e preparar os dados para classificação digital ou interpretação visual. O sucesso da detecção das superfícies de água parece mais dependente dos seguintes fatores: (1) especificações do sensor (comprimentos de onda e polarizações); (2) critérios do projeto (unidade de mapeamento mínimo e detalhe das categorias mapeadas); e (3) características ambientais (função de transferência de modulação). Assim, técnicas avançadas de análise de imagens podem não ser críticas, pois abordagens 
simples como o emprego de valores limites parecem comparáveis aos resultados a métodos mais complexos.

Outro atributo muito explorado na detecção das áreas inundáveis são os modelos digitais de elevação (MDE) oriundo de sensores remotos, por exemplo Interferometria SAR, Lidar e fotogrametria digital. Os DEMs têm sido amplamente utilizados para auxiliar no mapeamento e modelagem de inundação (CHEN et al., 2014; GRIMALDI et al., 2016). Os dados do DEM podem ser utilizados na correção dos pixels erroneamente classificados como água, considerando a declividade do terreno e a distância do rio (Huang et al., 2014). Além disso, séries temporais de DEM podem ser obtidos a partir de vários sensores espaciais (por exemplo, estéreo satélite ASTER, SPOT, Pleiades, WorldView, QuickBird e IKONOS; interferometria de radar SRTM, TanDEM-X e Sentinel-1).

Complementarmente, deve-se salientar que diversos algoritmos e ferramentas on-line foram desenvolvidos para a detecção e análise de inundações a partir de imagens de satélite, tais como: Observatório de Inundação de Dartmouth (Dartmouth Flood Observatory) (BRAKENRIDGE e ANDERSON, 2006), o Sistema Global de Detecção de Inundações (Global Flood Monitoring System) (KUGLER e DE GROEVE, 2007) e o Sistema de Monitoramento Global de Inundações (Global Flood Monitoring System) (WU et al., 2014). O Observatório de Inundações de Dartmouth disponibiliza gratuitamente a cobertura global dos grandes eventos de enchentes (1985 - presente) a partir do processamento de imagens MOSIS em seu site (http://floodobservatory.colorado.edu). O Sistema Global de Detecção de Inundações desenvolvido pela Joint Research Centre (JRC) da Comissão Europeia modifica e aprimora a metodologia desenvolvida pelo Observatório de Inundações de Dartmouth para o monitoramento de rios e detecção de áreas inundáveis utilizando imagens de radar do sensor “Advanced Microwave Scanning Radiometer for EOS” (AMSR-E), estando integrado ao "Global Disaster Alert and Coordination System" (www.gdacs.org/floods). Finalmente, o Sistema de Monitoramento Global de Inundações foi desenvolvido pela Universidade de Maryland com financiamento da NASA que utiliza a informação de precipitação em tempo real do "TRMM Multi-satellite Precipitation Analysis" (TMPA) para a modelagem do escoamento hidrológico. 


\section{COMPUTAÇÃO EM NUVEM - GOOGLE EARTH ENGINE}

O advento da computação em nuvem (Cloud Computing) traz profundas transformações no mundo da tecnologia da informação. A definição desse termo tem sido amplamente discutida (VAQUERO et al., 2009). Conforme Zhang et al. (2010), a dificuldade da definição desse termo é que diferentemente de outros termos técnicos não se trata de uma nova tecnologia, mas sim um novo modelo de operações que reúne um conjunto de tecnologias existentes para executar negócios de uma maneira diferente. Uma definição amplamente aceita do termo computação em nuvem é estabelecida pela National Institute of Standards and Technology (NIST) como um "modelo para permitir acesso de rede conveniente e sob demanda a um conjunto compartilhado de recursos de computação configuráveis (por exemplo, redes, servidores, armazenamento, aplicativos e serviços) que podem ser rapidamente provisionados e liberados com o mínimo esforço de gerenciamento ou interação de serviço fornecedor". Portanto, esse novo modelo proveniente da convergência de diferentes tecnologias permite um acesso de grande quantidade de dados, aplicações e serviços a partir de qualquer lugar com boa conexão de internet, fornecendo uma maximização, mobilidade e flexibilidade dos recursos computacionais aos usuários. Essa nova abordagem computacional possibilita um rápido compartilhamento do conhecimento e capacidade de resguardar os dados através de cópias de segurança, evitando perdas de informações.

Recentemente, foi lançado o sistema Google Earth Engine (https://earthengine.google.com/) baseado em computação em nuvem de dados geoespaciais e de sensoriamento remoto. Esse sistema é composto por conjuntos de imagens catalogados e prontos para o processamento com vários petabytes a partir de um serviço de computação paralela de alto desempenho, que permite o acesso do usuário por uma interface de programação de aplicativos (API) acessível pela Internet e um ambiente de desenvolvimento interativo (IDE) associado à Web (GORELICK et al., 2017). O sistema de processamento paralelo subdivide e distribui os cálculos automaticamente, estabelecendo agilidade de processamento. O sistema disponibiliza um grande acervo de coleções de imagens publicamente disponíveis, tais como: Landsat, Sentinel (Sentinel 1A/B, Sentinel 2A MSI), Moderate Resolution Imaging Spectroradiometer (MODIS), Advanced Very High Resolution Radiometer (AVHRR), Advanced Spaceborne Thermal Emission and Reflection Radiameter (ASTER), dados de topografia (por exemplo: Shuttle Radar Topography Mission, USGS National Elevation Dataset, USGS GMTED2010, GTOPO30 e ETOPO1), imagens e dados 
climáticos (por exemplo: TRMM 3B42 precipitation, Global precipitation measurement, CHIRPS precipitation, WorldClim), entre outros. As imagens são disponibilizadas com préprocessamento que facilita o uso imediato dos dados. A plataforma dispõe de um conjunto de algoritmos para seleção de imagens a partir de critérios específicos espaciais, espectrais, temporais ou outros. Além disso, essa ferramenta permite a criação e disseminação de novos algoritmos, que favorece uma célere evolução do conhecimento, visualização e disponibilização rápida de seus resultados.

Entre as várias aplicações da computação em nuvem para o processamento de grandes conjuntos de imagens de satélite, alguns são direcionados para o mapeamento da água superficial da Terra e de sua dinâmica (COLTIN et al., 2016; DELANCEY et al., 2017; HIRD et al., 2017; PEKEL et al., 2016; YAMAZAKI et al., 2016). A pesquisa desenvolvida por Pekel et al. (2016) quantifica a cobertura e mudanças da água superficial global nos últimos 32 anos (1984-2015) a partir de três milhões de imagens de satélite Landsat, com resolução espacial de 30 metros. A presença de água foi registrada de acordo com os meses e anos, evidenciando as localidades com permanência ou com mudanças sazonais. Apesar da magnitude da pesquisa, ainda persistem algumas lacunas tais como: falta de dados que afetam a análise sazonal, a resolução espacial de 30 metros impede a aplicação em corpos de água menores, áreas úmidas abaixo de vegetações não são detectadas e o ciclo de repetição de 16 dias pode ausentar eventos importantes de inundações.

\section{NANOSSATÉLITES}

O grande avanço tecnológico na construção de satélites de observação da Terra está na sua progressiva redução de tamanho e massa por meio da miniaturização dos componentes dos sensores, processadores e outros componentes eletrônicos de alto desempenho (SANDAU et al., 2010). Os satélites miniaturizados apresentam peso inferior que $500 \mathrm{~kg}$, sendo classificados como: minissatélites (100-500 kg), microssatélite (10-100 kg), nanossatélite (110kg), picossatélite (>1kg) e femtossatélites (10-100g) (SWEETING, 1992). Quanto menor o satélite, menor é o custo, tempo de construção e resposta. Os custos são ainda menores considerando orbitas de altitudes mais baixas (SHAO et al., 2014). Dessa forma, observa-se recentemente um alto crescimento de lançamento de satélites miniaturizados para diferentes tipos de estudos (CAPPELLETTI et al., 2018; CHUNG et al., 2016). Além disso, esses satélites oferecem uma oportunidade única para o estabelecimento de constelações com 
aquisições de dados de imagens em ciclos temporais mais curtos, que favorece $\mathrm{o}$ monitoramento de fenômenos físicos e desastres naturais.

Na observação da Terra, a principal empresa de desenvolvimento de nanossatélite é Planet Labs Inc. sediada em São Francisco, Califórnia. Essa empresa possui em órbita uma constelação de nanossatélites, que obtêm dados diários da superfície terrestre em RGB e infravermelho próximo. Os nanossatélites (denominados de Dove) apresentam uma dimensão de $10 \mathrm{~cm} \times 10 \mathrm{~cm}$ x $30 \mathrm{~cm}$ e um peso de $5 \mathrm{~kg}$. Essas dimensões são muito inferiores que um satélite tradicional de observação da Terra. Em comparação, o satélite Landsat 8 possui um diâmetro de 2,4 metros, comprimento de 3 metros e pesa aproximadamente 2 toneladas. Portanto, os nanossatélites da Planet Labs pesam 50 vezes menos do que o satélite Landsat 8 . Além disso, as imagens adquiridas com os nanossatélite possui uma resolução espacial de 3 a 5 metros contra a imagem do OLI-Landsat 8 de 30 metros. Essa dimensão reduzida possibilitou a empresa Planet Labs lançar 88 satélites no dia 15 de fevereiro de 2017 e atingir a maior frota de satélites em um único lançamento. Somando-se com os nanossatelites que já estavam em órbita foi alcançado um número total de 149 satélites em operação. A separação dos nanossatélites em torno de seus planos de órbita utilizou arraste diferencial devido a inexistência de propulsão a bordo, levando cerca de seis meses para o correto posicionamento após o lançamento (FOSTER et al., 2015, 2018). A constelação de satélites com visão nadir e órbita sincrônico ao sol alinhou-se em um único plano orbital para o imageamento diário de toda a Terra (FOSTER et al., 2018). Essa conformação de satélites uniformemente espaçados também permitiu o uso otimizado de estações terrestres disponíveis para capturar a imensa quantidade de imagens (11 TB dia), o que torna essa configuração preferida em relação às distribuições aleatórias de satélites ao longo da órbita (SWEETING, 2018).

A Figura 2 apresenta a imagens Planet do rio Parnaíba na região que margeia a cidade de Teresina (Piauí), considerando a época de cheia e de estiagem. A alta resolução espacial permite comparar os dois períodos evidenciando claramente as mudanças.

\section{CONSIDERAÇÕES FINAIS}

O processamento digital de imagens de sensoriamento remoto vem sendo amplamente utilizado para a detecção de mudanças dos corpos hídricos por fornecer informações sinópticas e repetitivas. Nas duas últimas décadas, houve um grande avanço de estudos a partir de dados de imagens orbitais para análise das áreas inundáveis, dinâmica fluvial, e previsão de riscos de inundação. No entanto, o advento das constelações de 
pequenos satélites com alto ciclo de revisita acopladas à internet, armazenamento em nuvem, novos algoritmos de processamento e alta disponibilidade de dados livres trazem rápidas mudanças e novas perspectivas do emprego do sensoriamento remoto para estudos geomorfológicos e hidrológicos. Esse arsenal tecnológico resulta em informações mais acuradas e em uma convergência de esforços para um monitoramento em escala global com alta frequência temporal.
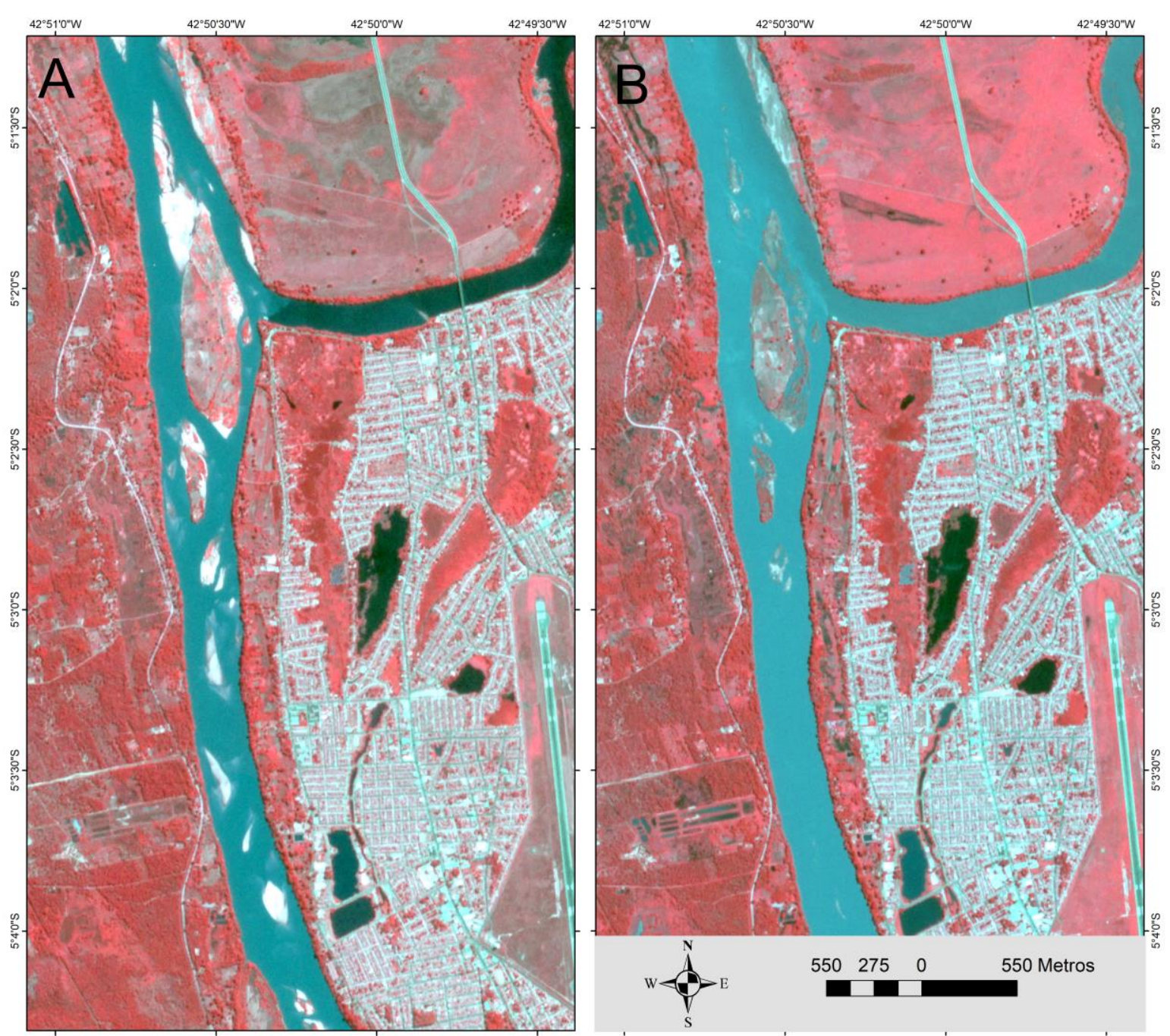

Figura 2. Imagens do nanossatélite Planet do rio Parnaíba na cidade de Teresina (Piauí). As imagens correspondem a um período com o rio baixo (02/07/2018), evidenciando os bancos de areia, e um período de cheia (24/04/2018). 


\section{REFERÊNCIAS}

AUGUSTEIJN, M. F.; WARRENDER, C. E. Wetland classification using optical and radar data and neural network classification. Int. J. Remote Sens., v. 19, n. 8, p. 1545-1560, 1998. BETBEDER, J.; RAPINEL, S.; CORPETTI, T.; POTTIER, E.; CORGNE, S.; HUBERTMOY, L. Multitemporal classification of TerraSAR-X data for wetland vegetation mapping. J. Appl. Remote Sens., v. 8, n. 1, p. 083648, 2014.

BOSCHETTI, M.; NUTINI, F.; MANFRON, G.; BRIVIO, P. A.; NELSON, A. (2014). Comparative analysis of normalised difference spectral indices derived from MODIS for detecting surface water in flooded rice cropping systems. PloS One, v. 9, n. 2, p. e88741, 2014.

BRAKENRIDGE, R.; ANDERSON, E. MODIS-based flood detection, mapping and measurement: the potential for operational hydrological applications. In: MARSALEK J.; STANCALIE, G.; BALINT, G. (Eds.). Transboundary floods: reducing risks through flood management. Dordrecht: Springer, 2006. p. 1-12. (Nato Science Series: IV: Earth and Environmental Sciences, v. 72).

CAPPELlETTI, C.; BATTISTINI, S.; GRAZIANI, F. Small launch platforms for microsatellites. Adv. Space Res., 2018 doi: 10.1016/j.asr.2018.05.004.

CARVAlHO JUNIOR, O. A.; BERBET, M. L. C.; MARTINS, E. S.; GUIMARÃES, R. F.; GOMES, R. A. T. Ambientes Cársticos. In: FlORENZANO, T. G. (Org.). Geomorfologia conceitos e tecnologias atuais. 1ed. São Paulo: Oficina de Textos, 2008, v. 1, p. 185-218.

CARVAlHO JÚNIOR, O. A.; COUTO JUNIOR, A. F.; DA SILVA, N. C.; DE SOUZA MARTINS, E.; CARVAlHO, A. P. F.; GOMES, R. A. T. Avaliação dos classificadores espectrais de mínima distância euclidiana e spectral correlation mapper em séries temporais NDVI-MODIS no campo de instrução militar de Formosa (GO). Revista Brasileira de Cartografia, v. 61, p. 399-412, 2009.

CARVALHO JÚNIOR, O. A.; GUIMARÃES, R. F.; MONTGOMERY, D. R.; GILLESPIE, A. R.; TRANCOSO GOMES, R. A.; DE SOUZA MARTINS, É.; SILVA, N. C. (2013). Karst depression detection using ASTER, ALOS/PRISM and SRTM-derived digital elevation models in the Bambuí Group, Brazil. Remote Sens., v. 6, n. 1, p. 330-351, 2013.

CHAUHAN, S.; SRIVASTAVA, H. S. Comparative evaluation of the sensitivity of multipolarised sar and optical data for various land cover. Int. J. Adv. Remote Sens. Gis Geogr. 2016, v. 4, p. 1-14. 
CHEN, Y.; HUANG, C.; TICEHURST, C.; MERRIN, L.; THEW, P. An evaluation of MODIS daily and 8-day composite products for floodplain and wetland inundation mapping. Wetlands, v. 33, p. 823-835, 2013.

CHEN, Y.; WANG, B.; POLlinO, C. A.; CUDDY, S. M.; MERRIN, L. E.; HUANG, C. Estimate of flood inundation and retention on wetlands using remote sensing and GIS. Ecohydrology, v. 7, n. 5, p. 1412-1420, 2014.

COLTIN, B.; MCMICHAEL, S.; SMITH, T.; FONG, T. (2016). Automatic boosted flood mapping from satellite data. Int. J. Remote Sens., v. 37, n. 5, p. 993-1015, 2016.

DELANCEY, E. R.; KARIYEVA, J.; CRANSTON, J.; BRISCO, B. Monitoring hydro temporal variability in Alberta, Canada with multi-temporal Sentinel-1 SAR data. Can. J. Remote Sens., v. 44, n. 1, p. 1-10, 2018.

DI BALDASSARRE, G.; SCHUMANN, G.; BRANDIMARTE, L.; BATES, P. Timely low resolution SAR imagery to support floodplain modelling: a case study review. Surv. Geophys., v. 32, n. 3, p. 255-269, 2011.

EVANS, T. L.; COSTA, M. P. F.; TELMER, K. H.; SILVA, T. S. F. Using ALOS/PALSAR and RADARSAT-2 to map land cover and seasonal inundation in the Brazilian Pantanal. IEEE J. Sel. Top. Appl., v. 3, p. 560-575, 2010.

FENG, L.; HU, C.; CHEN, X.; CAI, X.; TIAN, L.; GAN, W. Assessment of inundation changes of Poyang Lake using MODIS observations between 2000 and 2010. Remote Sens. Environ., v. 121, p. 80-92, 2012.

FOSTER, C.; HALLAM, H.; MASON, J. Orbit determination and differential-drag control of planet labs cubesat constellations. In: AAS/AIAA ASTRODYNAMICS SPECIALIST CONFERENCE, 2015, Vail, CO, USA. Proceeding..., Vail, CO: AAS Space Flight Mechanics Committee and the AIAA Astrodynamics Technical Committee, 2015, p. 1-13.

FOSTER, C.; MASON, J.; VITTALDEV, V.; LEUNG, L.; BEUKELAERS, V.; STEPAN, L.; ZIMMERMAN, R. Differential Drag Control Scheme for Large Constellation of Planet Satellites and On-Orbit Results. In: INTERNATIONAL WORKSHOP ON SATELLITE CONSTELLATIONS AND FORMATION FLYING, 9.; 2017, Boulder, CO, USA. Proceeding..., 2018, p. 1-18.

GALLANT, A. L. The Challenges of Remote Monitoring of Wetlands. Remote Sens., v. 7, p. 10938-10950, 2015.

GAO, B.C. NDWI-A normalized difference water index for remote sensing of vegetation liquid water from space. Remote Sens. Environ., v. 58, n. 3, p. 257-266, 1996. 
GILlESPIE, T. W.; CHU, J.; FRANKENBERG, E.; THOMAS, D. Assessment and prediction of natural hazards from satellite imagery. Prog. Phys. Geog., v. 31, n. 5, p. 459470, 2007.

GRIMALDI, S.; LI, Y.; PAUWELS, V. R.; WALKER, J. P. Remote sensing-derived water extent and level to constrain hydraulic flood forecasting models: opportunities and challenges. Surv. Geophys., v. 37, n. 5, p. 977-1034, 2016.

GUERSCHMAN, J.P.; WARREN, G.; BYRNE, G.; LYMBURNER, L.; MUELLER, N.; VAN-DIJK, A. MODIS-Based Standing Water Detection for Flood and Large Reservoir Mapping: Algorithm Development and Applications for the Australian Continent. Canberra, Australia: CSIRO, 2011.

GUIMARÃES, R. F.; CARVALHO JUNIOR, O. A.; GOMES, R. A. T.; FERNANDES, N. F. Movimentos de Massa. In: FlorenZANO, T. G. (Org.). Geomorfologia conceitos e tecnologias atuais. 1 ed. São Paulo: Oficina de Textos, 2008, v. 1, p. 159-184.

HALL, A. C.; SCHUMANN, G. J. P.; BAMBER, J. L.; BATES, P. D. Tracking water level changes of the Amazon Basin with space-borne remote sensing and integration with large scale hydrodynamic modelling: A review. Phys. Chem. Earth, Parts A/B/C, v. 36, n. 7-8, p. 223-231, 2011.

HENDERSON, F. M.; LEWIS, A. J. Radar detection of wetland ecosystems: a review. Int. J. Remote Sens., v. 29, n. 20, p. 5809-5835, 2008.

HERMAS, E.; LEPRINCE, S.; EL-MAGD, I. A. Retrieving sand dune movements using subpixel correlation of multi-temporal optical remote sensing imagery, northwest Sinai Peninsula, Egypt. Remote Sens. Environ., v. 121, p. 51-60, 2012.

HESS, L.; MELACK, J.; SIMONETT, D. Radar detection of flooding beneath the forest canopy: a review. Int. J. Remote Sens., v. 11, p. 1313-1325, 1990.

HIRD, J. N.; DELANCEY, E. R.; MCDERMID, G. J.; KARIYEVA, J. Google Earth Engine, open-access satellite data, and machine learning in support of large-area probabilistic wetland mapping. Remote Sens., v. 9, n. 12, p. 1315, 2017.

HU, C. A novel ocean color index to detect floating algae in the global oceans. Remote Sens. Environ., v. 113, n. 10, p. 2118-2129, 2009.

HUANG, C.; CHEN, Y.; WU, J. DEM-based modification of pixel-swapping algorithm for enhancing floodplain inundation mapping. Int. J. Remote Sens., v. 35, n. 1, p. 365-381, 2014. 
HUETE, A.; DIDAN, K.; MIURA, T.; RODRIGUEZ, E. P.; GAO, X.; FERREIRA, L. G. Overview of the radiometric and biophysical performance of the MODIS vegetation indices. Remote Sens. Environ., v. 83, n. 1-2, p. 195-213, 2002.

HUGENHOLTZ, C. H.; LEVIN, N.; BARCHYN, T. E.; BADDOCK, M. C. Remote sensing and spatial analysis of aeolian sand dunes: A review and outlook. Earth-Sci. Rev., v. 111, n. 3-4, p. 319-334, 2012.

ISLAM, A. S.; BALA, S. K.; HAQUE, M. A. (2010). Flood inundation map of Bangladesh using MODIS time-series images. J. Flood Risk Manag., v. 3, n. 3, p. 210-222, 2010.

JI, L.; ZHANG, L.; WYLIE, B. Analysis of dynamic thresholds for the normalized difference water index. Photogram. Eng. Remote Sens. 2009, 75, 1307-1317.

JIANG, Z.; QI, J.; SU, S.; ZHANG, Z.; WU, J. Water body delineation using index composition and HIS transformation. Int. J. Remote Sens., v. 33, n. 11, p. 3402-3421, 2012. JONES, K.; LANTHIER, Y.; VAN DER VOET, P.; VAN VALKENGOED, E.; TAYLOR, D.; FERNÁNDEZ-PRIETO, D. Monitoring and assessment of wetlands using Earth Observation: The GlobWetland project. J. Environ. Manage., v. 90, n. 7, p. 2154-2169, 2009.

KÄÄB, A. Remote sensing of permafrost-related problems and hazards. Permafrost Periglac., v. 19, n. 2, p. 107-136, 2008.

KASISCHKE, E.; MELACK, J. and DOBSON, M. The use of radars for ecological applications: a review. Remote Sens. Environ., v. 59, p. 141-156, 1997.

KIM, W. Cloud computing architecture. Int. J. Web Grid Serv, v. 9, n. 3, p. 287-303, 2013.

KLEMAS, V. Remote sensing of floods and flood-prone areas: an overview. J. Coastal Res., v. 31, n. 4, p. 1005-1013, 2014.

KUGLER, Z.; DE GROEVE, T. The global flood detection system. Luxembourg: Office for Official Publications of the European Communities, 2007, 1-45 p. (JRC scientific and technical reports).

LANE, S. N.; WESTAWAY, R. M.; MURRAY HICKS, D. Estimation of erosion and deposition volumes in a large, gravel-bed, braided river using synoptic remote sensing. Earth Surf. Proc. Land., v. 28, n. 3, p. 249-271, 2003.

LI, W.; DU, Z.; LING, F.; ZHOU, D.; WANG, H.; GUI, Y.; SUN, B.; ZHANG, X. A comparison of land surface water mapping using the normalized difference water index from TM, ETM+ and ALI. Remote Sens., v. 5, n. 11, p. 5530-5549, 2013. 
MATA, C. L.; DE CARVALHO JÚNIOR, O. A.; DE CARVALHO, A. P. F.; GOMES, R. A. T.; DE SOUZA MARTINS, É.; GUIMARÃES, R. F. (2007). Avaliação multitemporal da susceptibilidade erosiva na bacia do Rio Urucuia (MG) por meio da equação universal de perda de solos. Revista Brasileira de Geomorfologia, v. 8, n. 2, 2007.

MCFEETERS, S. K. The use of the Normalized Difference Water Index (NDWI) in the delineation of open water features. Int. J. Remote Sens., v. 17, n. 7, p. 1425-1432, 1996.

MELACK, J. M.; HESS, L. L. Remote sensing of the distribution and extent of wetlands in the Amazon Basin, In: JUNK, W. J.; PIEDADE, M. T. F.; WITTMANN, F.; SCHÖNGART, J.; PAROLIN, P. (Eds.). Amazonian Floodplain Forests: Ecophysiology, Biodiversity and Sustainable Management. 1 ed. Berlin, Germany: Springer, 2010, p. 43-59.

METTERNICHT, G.; HURNI, L.; GOGU, R. Remote sensing of landslides: An analysis of the potential contribution to geo-spatial systems for hazard assessment in mountainous environments. Remote Sens. Environ., v. 98, n. 2-3, p. 284-303, 2005.

MUSA, Z. N.; POPESCU, I.; MYNETT, A. A review of applications of satellite SAR, optical, altimetry and DEM data for surface water modelling, mapping and parameter estimation. Hydrol. Earth Syst. Sc., v. 19, n. 9, p. 3755-3769, 2015.

OUMA, Y. O.; TATEISHI, R. A water index for rapid mapping of shoreline changes of five East African Rift Valley lakes: An empirical analysis using Landsat TM and ETM+ data. Int. J. Remote Sens., 27, 3153-31812006.

OZESMI, S. L.; BAUER, M. E. Satellite remote sensing of wetlands. Wetl. Ecol. Manag., v. 10, n. 5, p. 381-402, 2002.

PARMUCHI, M. G.; KARSZENBAUM, H.; KANDUS, P. Mapping wetlands using multitemporal RADARSAT-1 data and a decision-based classifier. Can. J. Remote Sens., v. 28, n. 2, p. 175-186, 2002.

PEKEL, J. F.; COTTAM, A.; GORELICK, N.; BELWARD, A. S. (2016). High-resolution mapping of global surface water and its long-term changes. Nature, v. 540, n. 7633, p. 418, 2016.

PEKEL, J. F.; VANCUTSEM, C.; BASTIN, L.; CLERICI, M.; VANBOGAERT, E.; BARTHOLOMÉ, E.; DEFOURNY, P. A near real-time water surface detection method based on HSV transformation of MODIS multi-spectral time series data. Remote Sens. Environ., v. 140, p. 704-716, 2014.

RAHMAN, M. S.; DI, L. The state of the art of spaceborne remote sensing in flood management. Nat. Hazards, v. 85, n. 2, p. 1223-1248, 2017. 
RAMSEY III, E. Radar remote sensing of wetlands. In: LUNETTA, R.; ELVIDGE, C. (Eds.). Remote Sensing Change Detection: Environmental Monitoring Methods and Applications. Chelsea, MI: Ann Arbor Press, 1998, cap. 13. p. 211-243.

ROKNI, K.; AHMAD, A.; SELAMAT, A.; HAZINI, S. (2014). Water feature extraction and change detection using multitemporal Landsat imagery. Remote Sens., v. 6, n. 5, p. 4173 4189, 2014.

ROUSE, J. W.; HAAS, R. H.; SCHELL, J. A.; DEERING, D. W. Monitoring Vegetation Systems in the Great Plains with ERTS (Earth Resources Technology Satellite). In: EARTH RESOURCES TECHNOLOGY SATELLITE SYMPOSIUM, $3^{\text {th }}, 1973$, Greenbelt, ON, Canada, Proceedings... 1973; v. SP-351. p. 309-317.

SANDAU, R. Status and trends of small satellite missions for Earth observation. Acta Astronaut., v. 66, n. 1-2, p. 199-201, 2017.

SAWAYA, K. E.; OLMANSON, L. G.; HEINERT, N. J.; BREZONIK, P. L.; BAUER, M. E. Extending satellite remote sensing to local scales: land and water resource monitoring using high-resolution imagery. Remote Sens. Environ., v. 88, n. 1-2, p. 144-156, 2003.

SCAIONI, M.; LONGONI, L.; MELILlO, V.; PAPINI, M. Remote sensing for landslide investigations: An overview of recent achievements and perspectives. Remote Sens., v. 6, n. 10, p. 9600-9652, 2014.

SCHMULLIUS, C.; EVANS, D. Synthetic aperture radar (SAR) frequency and polarization requirements for applications in ecology, geology, hydrology, and oceanography: a tabular status quo after SIR-C/X-SAR. Int. J. Remote Sens., v. 18, p. 2713-2722, 1997.

SCHUMANN, G. J. P.; BATES, P. D.; NEAL, J. C.; ANDREADIS, K. M. Measuring and mapping flood processes. In: PARON, P.; DI BALDASSARRE, G. (Ed.). Hydrometeorological hazards, risks and disasters. Boston: Elsevier, 2015. cap. 2, p. 35-64.

SCHUMANN, G. J.-P.; MOLLER, D. K. Microwave remote sensing of flood inundation. Phys. Chem. Earth, Parts A/B/C, v. 83, p. 84-95, 2015.

SHAO, A.; WERTZ, J. R.; KOLTZ, E. A. Quantifying the cost reduction potential for Earth Observation Satellites. In: HATTON, S. (Ed.). Proceedings of the 12th Reinventing Space Conference. Cham: Springer, 2017. p. 199-210.

SHEN, L.; LI, C. Water Body Extraction from Landsat ETM+ Imagery Using Adaboost Algorithm. In: INTERNATIONAL CONFERENCE ON GEOINFORMATICS, $8^{\text {th }}, 2010$, Beijing, China, Proceedings... IEEE Geoscience and Remote Sensing Society, 2010. p. 1-4. 
SIART, C.; BUBENZER, O.; EITEL, B. Combining digital elevation data (SRTM/ASTER), high resolution satellite imagery (Quickbird) and GIS for geomorphological mapping: A multi-component case study on Mediterranean karst in Central Crete. Geomorphology, v. 112, p. 106-121, 2009.

Silva, N. C.; CARVAlho JUNIOR, O. A.; GUIMARÃES, R. F.; Silva, D. E. Geotecnologia e proteção ambiental: a detecção de mundanças para análises temporais do uso do solo a serviço dos historiadores ambientais. In: FRANCO, J. L. A.; DUTRA E SILVA, S.; DRUMMOND, J. A.; TAVARES, G. G. (Org.). História Ambiental: fronteiras, recursos naturais e conservação da natureza. 1ed. Rio de Janeiro: Garamond, 2012, v. 1, p. 79-96. SILVA, T. S. F.; COSTA, M. P. F.; MELACK, J. M. Spatial and temporal variability of macrophyte cover and productivity in the eastern Amazon floodplain: A remote sensing approach. Remote Sens. Environ., 114, 1998-2010, 2010.

SMITH, L. C. Satellite remote sensing of river inundation area, stage, and discharge: A review. Hydrol. Process., v. 11, n. 10, p. 1427-1439, 1997.

SWEETING, M. N. (2018). Modern Small Satellites-Changing the Economics of Space. Proceedings of the IEEE, v. 106, n. 3, p. 343-361, 2018.

SWEETING, M. N. UoSAT microsatellite missions. Electron. Commun. Eng., v. 4, n. 3, p. 141-150, 1992.

TICEHURST, C.; GUERSCHMAN, J. P.; CHEN, Y. The strengths and limitations in using the daily MODIS open water likelihood algorithm for identifying flood events. Remote Sens., v. 6, n. 12, p. 11791-11809, 2014.

TOFANI, V.; SEGONI, S.; AGOSTINI, A.; CATANI, F.; CASAGLI, N. Use of remote sensing for landslide studies in Europe. Nat. Hazards Earth Sys., v. 13, n. 2, p. 299-309, 2013.

TRALli, D. M.; BLOM, R. G.; ZLOTNICKI, V.; DONNELLAN, A.; EVANS, D. L. Satellite remote sensing of earthquake, volcano, flood, landslide and coastal inundation hazards. ISPRS J. Photogramm., v. 59, n. 4, p. 185-198, 2005.

VAN WESTEN, C. J. Remote sensing and GIS for natural hazards assessment and disaster risk management. Treatise on Geomorphology, v. 3, p. 259-298, 2013.

VAQUERO, L. M.; RODERO-MERINO, L.; CACERES, J.; LINDNER, M. A break in the clouds: towards a cloud definition. ACM SIGCOMM Computer Communication Review, v. 39, n. 1, p. 50-55, 2008. 
VRIELING, A. Satellite remote sensing for water erosion assessment: A review. Catena, v. 65, n. 1, p. 2-18, 2006.

WHITCOMB, J.; MOGHADDAM, M.; MCDONALD, K.; KELLNDORFER, J.; PODEST, E. Mapping vegetated wetlands of Alaska using L-band radar satellite imagery. Can. J. Remote Sens., v. 35, n. 1, p. 54-72. 2009

WILSON, E.H.; SADER, S.A. Detection of forest harvest type using multiple dates of Landsat TM imagery. Remote Sens. Environ., v. 80, p. 385-396, 2002.

WU, H.; ADLER, R. F.; TIAN, Y.; HUFFMAN, G. J.; LI, H.; WANG, J. Real-time global flood estimation using satellite-based precipitation and a coupled land surface and routing model. Water Resour. Res., v. 50, n. 3, p. 2693-2717, 2014.

XIAO, X.; BOLES, S.; FROLKING, S.; LI, C.; BABU, J. Y.; SALAS, W.; MOORE III, B. Mapping paddy rice agriculture in South and Southeast Asia using multi-temporal MODIS images. Remote Sens. Environ., v. 100, n. 1, p. 95-113, 2006.

XIAO, X.; ZHANG, Q.; BRASWELL, B.; URBANSKI, S.; BOLES, S.; WOFSY, S.C.; MOORE, B.; OJIMA, D. Modeling gross primary production of a deciduous broadleaf forest using satellite images and climate data. Remote Sens. Environ., v. 91, p. 256-270, 2004.

XU, H. Modification of Normalized Difference Water Index (NDWI) to enhance open water features in remotely sensed imagery. Int. J. Remote Sens., v. 27, n. 14, p. 3025-3033, 2006.

YAMAZAKI, D.; TRIGG, M.A. Hydrology: The dynamics of Earth's surface water. Nature, v. 540, p. 348-349, 2016.

YAN, K.; DI BALDASSARRE, G.; SOLOMATINE, D. P.; SCHUMANN, G. J. P. A review of low-cost space-borne data for flood modelling: topography, flood extent and water level. Hydrol. Process., v. 29, n. 15, p. 3368-3387, 2015.

ZHANG, Q.; CHENG, L.; BOUTABA, R. Cloud computing: state-of-the-art and research challenges. Journal of Internet Services and Applications, v. 1, n. 1, p. 7-18, 2010.

ZHANG, Y.; WANG, C.; WU, J.; QI, J.; SALAS, W. A. Mapping paddy rice with multitemporal ALOS/PALSAR imagery in southeast China. Int. J. Remote Sens., v. 30, n. 23, p. 6301-6315, 2009. 